\title{
Erratum to: Musculoskeletal Injury, Functional Disability, and Health-Related Quality of Life in Aging Mexican Immigrant Farmworkers
}

\author{
M. M. Weigel · R. X. Armijos · O. Beltran
}

Published online: 4 April 2013

(C) Springer Science+Business Media New York 2013

Erratum to: J Immigrant Minority Health

DOI 10.1007/s10903-013-9788-6

Authors of the paper have discovered that they missed one erroneous stray line $(-0.11-1.390 .17)$ under model 3 of Table 4 should be deleted. Correct table is given below:

The online version of the original article can be found under doi:10.1007/s10903-013-9788-6.

M. M. Weigel $(\bowtie) \cdot R$. X. Armijos

Department of Public Health Sciences, College of Health

Sciences, University of Texas at El Paso (UTEP),

El Paso, TX 79968, USA

e-mail: mmweigel@utep.edu

M. M. Weigel · R. X. Armijos · O. Beltran

Human Immunology and Disease Research Laboratory, UTEP

College of Health Sciences, El Paso, TX 79968, USA

M. M. Weigel · R. X. Armijos

UTEP Center for Interdisciplinary Health Research and Evaluation, UTEP College of Health Sciences,

El Paso, TX 79968, USA

O. Beltran

Interdisciplinary Health Science Doctoral Program, UTEP

College of Health Sciences and School of Nursing,

El Paso, TX 79968, USA 
Table 4 Regression analysis of current persistent musculoskeletal injury on the SF36 mental composite scale (MCS)

\begin{tabular}{|c|c|c|c|c|c|c|c|c|c|}
\hline \multirow[t]{2}{*}{ Variables } & \multicolumn{3}{|c|}{ Model 1} & \multicolumn{3}{|c|}{ Model 2} & \multicolumn{3}{|c|}{ Model 3} \\
\hline & $\beta$ & $\mathrm{t}$ & $p$ & $\beta$ & $\mathrm{t}$ & $p$ & $\beta$ & $\mathrm{t}$ & $p$ \\
\hline Musculoskeletal injury number & -0.31 & -4.32 & $<0.0001$ & -0.31 & -4.13 & $<0.0001$ & -0.27 & -3.36 & 0.001 \\
\hline Age (years) & & & & -0.49 & -0.59 & 0.56 & -0.60 & -0.69 & 0.49 \\
\hline Sex (female) & & & & 0.07 & 0.97 & 0.34 & 0.62 & 0.81 & 0.42 \\
\hline Education (years) & & & & 0.10 & 0.09 & 0.93 & -0.03 & -0.31 & 0.76 \\
\hline Diabetes & & & & & & & -0.11 & -1.39 & 0.17 \\
\hline Cardiovascular disease & & & & & & & 0.03 & 0.38 & 0.71 \\
\hline Body mass index & & & & & & & -0.11 & -1.44 & 0.15 \\
\hline Any treatment for PMI & & & & & & & 0.06 & 0.82 & 0.41 \\
\hline $\mathrm{R}^{2}$ & 0.09 & & & 0.09 & & & 0.09 & & \\
\hline
\end{tabular}

